\title{
La scénarisation des usages du territoire : des dispositifs participatifs aux logiques managériales et marketing
}

Immersive and Multimodal Participatory Devices: The Managerial and Marketing Dimensions of Public Debate

Caroline Didier

\section{CpenEdition}

Journals

Édition électronique

URL : http://journals.openedition.org/edc/8298

DOI : 10.4000/edc.8298

ISSN : 2101-0366

Éditeur

Université de Lille

Édition imprimée

Date de publication : 1 décembre 2018

Pagination : 189-206

ISBN : 978-2-917562-20-8

ISSN : 1270-6841

Référence électronique

Caroline Didier, « La scénarisation des usages du territoire : des dispositifs participatifs aux logiques managériales et marketing », Études de communication [En ligne], 51 | 2018, mis en ligne le 01 janvier 2021, consulté le 11 février 2021. URL : http://journals.openedition.org/edc/8298 ; DOI : https:// doi.org/10.4000/edc.8298 


\section{La scénarisation des usages}

du territoire : des dispositifs

participatifs aux logiques

managériales et marketing

Immersive and Multimodal Participatory

Devices: The Managerial and Marketing

Dimensions of Public Debate 
Afin de renouveler les formes du débat public, les institutions territoriales et culturelles s'orientent vers des formats participatifs plus visuels et théâtraux. De la balade urbaine au jeu de rôle, les dispositifs conçus autour de l'aménagement des territoires mettent l'usager à l'épreuve et sur scène face à un public. L'article souhaite éclairer les logiques managériales de ces modes tournés vers une participation collaborative, scénique et sensorielle, qui entrent en concurrence avec les objectifs d'élargissement et de mixité des publics. Cette recherche met en évidence les dimensions marketing de l'engagement corporel dans l'espace public, les contraintes de productivité de la participation et les effets de la scénarisation sur la conflictualité. Le suivi ethnographique de deux dispositifs permet de montrer comment ces logiques sont réinvesties en puissance expressive politique par les participants.

Mots-clés : dispositifs participatifs, débat public, management de la participation, formats créatifs, acteurs, publics, engagement corporel, politisation.
The participatory devices of cultural and local institutions are increasingly characterized by theatrical and visual formats. From the "urban stroll" to role playing, local authorities seek to renew traditional forms of public debate concerning territorial development. Participatory devices are becoming more immersive and multimodal, in an attempt to engage participants both physically and publicly. This article explores the managerial and marketing dimensions of these participatory frameworks which construct participation as a visible, collaborative and sensory experience. Multi-sited ethnography of two local communities reveals how participants redefine these frameworks as vehicles for political expression.

Keywords: participatory devices, public debate, participation management, creative formats, actors, publics, bodily engagement, politicization. 
Au sein des dispositifs participatifs des institutions territoriales et culturelles, des mises en scène des usages du territoire sont proposées en alternative aux formats traditionnels du débat public. Les collectivités locales s'associent de plus en plus à des lieux culturels afin de mettre en place des projets participatifs ayant un rayonnement à l'échelle métropolitaine. Des formats plus visuels et théâtraux sont mobilisés, forgés par le théâtre action et le jeu de rôle'. Les recherches ${ }^{2}$ sur les dispositifs participatifs démontrent qu'il existe une normativité dans les stratégies de mise en scène pour donner à voir le «bon usage " du territoire. Les deux dispositifs participatifs que nous avons suivis montrent comment ces formats sont guidés par des logiques managériales et marketing et comment celles-ci entrent en concurrence avec des objectifs d'élargissement et de mixité des publics.

Portés par des dirigeants de collectivités locales et de lieux culturels, les projets participatifs font appel à plusieurs types d'acteurs professionnels tels que des élus, des universitaires, des acteurs culturels, des urbanistes et des artistes. Si leur collaboration dans les projets participatifs pose «la question de la médiation quand le projet culturel se confond avec un projet de ville induisant une politisation de la fonction » (Da Lage, 2008, 2), elle pose également la question du management de la participation pouvant induire une dépolitisation du débat. Alors que la rhétorique participative "vise à créer de l'adhésion et du consensus "(Da Lage, 2008), ces formats particuliers semblent paradoxalement renforcer cette visée instrumentale en scénarisant la conflictualité. Alors comment saisir ce paradoxe dans des modes participatifs de plus en plus esthétisés? En outre, la mobilisation de formats visuels et scéniques pose, au même titre que pour le débat traditionnel, la question de l'accessibilité et de l'appropriation. Comment ces formats particuliers permettent-ils d'être investis par les participants?

Teintés d'un marketing territorial présent à l'échelon local depuis les lois de décentralisation (Pailliart, 1993), les dispositifs suivent une logique de mise en scène similaire aux marques. Cherchant à formaliser un discours plus tangible ${ }^{3}$ sur le territoire, les collectivités s'attachent à vouloir " co-construire une parole " illustrant le vécu des usagers. De cette manière, elles entendent agir sur l'effet de proximité (Le Bart et Lefebvre, 2005). Ce phénomène se traduit au niveau de la forme, avec des productions plus visuelles des participants à l'œuvre dans l'espace public.

1 Le théâtre action s'appuie sur les interactions entre la scène et la salle. Il est né en Belgique dans les années soixante et répond à un idéal de démocratisation culturelle, en visant à « donner la parole par la voix théâtrale à ceux qui ne l'ont pas, travailler à ce que des spectateurs de leur quotidien en deviennent les acteurs, au travers des projets de créations collectives, porter cette parole auprès de publics dit 'culturellement et socialement défavorisés' ", selon I'article de Mourin G. (2002).

2 Voir les travaux de Appel V., Bando C., Boulanger H. et al., (2008), Monnoyer-Smith L., $(2007$; 2006) et Bonaccorsi J., Julliard V., (2012).

3 Qui peut être perçu par les sens. 
Dans le cadre de notre recherche doctorale ${ }^{4}$, nous avons donc suivi et analysé deux dispositifs participatifs en particulier - les Ateliers Citoyens de Grenoble-Alpes Métropole et les Ateliers Arts-Sciences de la Scène nationale I'Hexagone de Meylan. Ces ateliers se sont déroulés chacun sur plus de six mois ${ }^{5}$. Le dispositif de la communauté d'agglomération était organisé par la chargée de mission Prospective et participation des habitants, en lien avec des élus et des personnes des services techniques. Celui de la structure culturelle était piloté par une équipe regroupant son directeur, certains de ses cadres, des urbanistes, des artistes et des enseignants chercheurs (en géographie, philosophie et communication).

Plusieurs points permettaient d'établir une comparaison entre les deux dispositifs. Tout d'abord, les sujets portaient sur les usages du territoire : "l'eau comme ressource et comme risque " pour la Métro, et "le circuit-court dans l'agglomération » pour la structure culturelle. Les deux dispositifs ont impliqué des partenariats avec des chercheurs et des spécialistes. Ensuite, les ateliers ont donné lieu à une restitution collective ${ }^{6}$ sur scène avec du jeu de rôle, de la danse ainsi que la diffusion de vidéos et de photos montrant les participants à la découverte du territoire. Enfin, les participants ont été sélectionnés auprès de publics captifs des structures et contactés par mail. Certains participants étaient contraints d'assister à ces ateliers. Pour l'Atelier Métro Citoyen (AMC), sur la dizaine de participants, la moitié y assistait en tant que représentants associatifs et non par intérêt personnel. Pour l'institution culturelle, sur environ quatre-vingt participants, une quarantaine d'étudiants devaient y participer dans le cadre d'un cours. Un nombre important d'abandons a par conséquent pu être noté?.

Afin de caractériser la participation, nous avons fait de l'observation participante avec une démarche ethnographique (Berger, 2008), en co-animant des ateliers. Notre grille de recueil de données s'est appuyée sur plusieurs indicateurs : la capacité ${ }^{8}$ des participants dans l'action à dépasser les consignes,

4 Didier C., (2015). Enjeux communicationnels et injonctions à la créativité dans des dispositifs participatifs, thèse de doctorat, Université Grenoble Alpes.

5 Entre octobre 2011 et juillet 2013.

6 Pour les Ateliers Citoyens de Grenoble-Alpes Métropole, la restitution collective a eu lieu le 29 mai 2012. Pour l'Atelier de l'Imaginaire du Festival ArtsSciences de la Scène nationale l'Hexagone de Meylan, la restitution collective a eu lieu le 15 janvier 2013.

7 Les plus investis représentaient deux catégories principales : des retraités et des précaires (demandeurs d'emploi, personnes en reconversion professionnelle et étudiants en fin de cycle). L'investissement plus important de ces personnes peut trouver une explication dans la sociabilité créée par les ateliers. Ces participants trouvent l'expérience valorisante car elle donne ensuite la possibilité de pouvoir discuter du sujet entre amis, de gagner de la confiance en soi pour des présentations professionnelles ou d'être plus convaincant aux entretiens d'embauche.

8 Nous nous appuyons sur l'acception élargie de Paul Ricoeur, $(2005,215)$ : "Un pont sera jeté entre les formes individuelles de capacités et des formes 
la capacité des participants après l'action à s'interroger sur le bien-fondé de la démarche et la capacité de redéfinition de la participation à partir des difficultés rencontrées. L'observation participante a été approfondie par des entretiens ${ }^{9}$ semi-directifs avec les organisateurs et les participants, sur lesquels nous allons nous appuyer afin d'analyser les enjeux de l'intérêt des collectivités locales pour ces dispositifs. Ils ont été interrogés sur le sens des mises en scène, avec photomontages, vidéos, écritures, lectures, chorégraphies et jeux de rôle.

Nous nous centrons sur la mise en scène des engagements dans l'espace public (Goffman, 1973, 1987), sur les contraintes de production et sur les conséquences de la scénarisation en tant que " performance » (Dewey, 2010). II est question pour nous de saisir à la fois le sens de l'engagement physique sur le territoire et sa performativité (Caune, 1981). Notre étude a permis de valider que la forme oriente la manière dont des participants conçoivent l'expérience en conformité ou en non-conformité avec les consignes d'être créatif ${ }^{10}$. Des consignes qui sont puisées dans les outils du management et qui ont pu entrer en concurrence avec les compétences artistiques et scéniques dont les participants devaient faire preuve pour exprimer leur point de vue.

Nous nous attacherons dans ce texte à présenter les différentes logiques qui poussent les collectivités locales à développer ces dispositifs souscrivant au registre de la monstration. Pour ce faire, nous présenterons dans un premier temps les modalités de l'engagement défini par les dispositifs. Nous verrons ensuite le cadre théorique des études théâtrales adaptées pour analyser la performativité de l'engagement corporel au sein des dispositifs. Puis il s'agira de montrer au travers d'un conflit que ces modalités répondent à un objectif de communication visant à faire consensus. Enfin, nous conclurons sur ces procédés de la communication publique renouvelés qui instrumentalisent la participation et tendent vers la représentation, mais qui sont réinvestis par les participants à des fins collectives ou personnelles.

sociales susceptibles de faire transition entre la reconnaissance de soi et la reconnaissance mutuelle. Dans cette acception élargie, les capacités en question ne sont plus seulement attestées par des individus, mais revendiquées par des collectivités et soumises à l'appréciation et à l'approbation publiques".

9 Sur la période de juillet 2012 à janvier 2014.

10 Voici un extrait de la consigne distribuée aux participants lors de l'atelier sur le circuit-court avec « les règles de la créativité » sous l'acronyme "T.C.Q.F.D. » : " T comme Tout est bon à dire. C comme Censure et autocensure abolie [...]. Q comme Quantité et non pas qualité. F comme Folles, Fantaisistes, Farfelues. Toutes les idées, même les plus extravagantes, les plus choquantes, sont les bienvenues. D comme Détournement. II faut jouer avec les idées, les reprendre, les déformer, les associer avec d'autres ". En étant une injonction paradoxale, la consigne a entrainé la confusion : la créativité n'est pas convoquée par les organisateurs pour mieux débattre d'un problème. Elle est mobilisée dans l'espoir d'attirer plus de monde aux ateliers et des publics diversifiés. 


\section{1. \\ Modalités d'une participation visible et collaborative}

Afin de dynamiser les formes du débat public, les professionnels de la participation font appel à des comédiens et des artistes pour réaliser des animations et des mises en scène avec les habitants sur le territoire. Les activités sont encadrées par des animateurs ${ }^{11}$ qui sont des professionnels sans formation d'animation et issus d'horizons sociaux plutôt élevés : des cadres de la participation, de l'enseignement, ou de l'action culturelle. Ils sont partenaires ou salariés des structures organisatrices, et interviennent sans cadre bien circonscrit en amont de leur participation ${ }^{12}$. L'animation sur le terrain constitue une alternative à la discussion en lieu clos, les débats publics étant jugés trop techniques et élitistes pour le public, comme veulent le croire les promoteurs de la participation.

Se mettre en scène sur le terrain constitue de plus en plus une condition d'actualisation des dispositifs participatifs. Il y a dix ans, la chercheuse en science politique Magali Nonjon remarquait déjà des pratiques d'animation croissantes sur le territoire afin de faciliter la « démocratie locale». Selon elle, cette multiplication traduisait une volonté de toucher plus de publics en se rapprochant du terrain :

Les animateurs de ces ateliers, convaincus de l'inégalité des individus devant la prise de parole en public, manifestent une certaine critique vis-à-vis des dispositifs classiques de concertation, dont le seul support reste la réunion publique. Pour accéder à la réalité, il faut 'mettre en scène le terrain' mais aussi 'se mettre en scène' sur le terrain (Nonjon, 2005).

Il faut montrer que le participant « pratique » son territoire, qu'il l'éprouve physiquement. Les actions doivent attirer l'attention sur la dimension physique de l'engagement dans l'espace public. Il peut s'agir par exemple d'interagir avec des passants dans des zones urbaines très fréquentées en tentant de provoquer un contact. C'est ainsi que pour l'Atelier de l'Imaginaire porté par l'institution

11 Pour l'atelier de la Métro, un groupe d'une dizaine de personnes encadré par la chargée de mission Prospective et participation des habitants. Elle s'est entourée d'universitaires (géographe, chercheur en communication). Pour l'institution culturelle, une dizaine de groupes encadrés par des cadres de la structure, des enseignants chercheurs co-pilotant le projet (philosophe, géographe, chercheur en communication), ou des membres d'un collectif d'artistes associé (travaillant sur des balades urbaines en particulier).

12 Nous emploierons le terme de " concepteur/animateur » tiré de la typologie de l'article « Médiation culturelle : I'enjeu de la gestion des ressources humaines " pour montrer la tension qui existe au sein d'une même fonction entre plusieurs postes (chargé de projet, animateur, médiateur, communicant, artiste) et plusieurs domaines professionnels (la communication publique, la médiation culturelle et l'animation théâtrale). 
culturelle, un groupe ${ }^{13}$ a filmé dans une gare la réaction de passants à qui il offrait une fleur sans dire un mot. Un autre groupe a proposé un parcours qui consistait à emprunter cinq moyens de transport dans l'agglomération sans prendre sa voiture (bulles, bus, marche, train et co-voiturage). Les groupes se soumettent ainsi à des exercices de mise en situation, aux allures parfois de jeux de piste, afin de suggérer différentes manières de poser le problème. Ils arpentent le terrain en quête de "curiosités territoriales ${ }^{14}$ afin d'illustrer un propos sur les usages.

Il est complexe d'envisager les tenants de la longévité de ces ateliers. En les associant toujours actuellement à de l'expérimentation, les praticiens rencontrés ne démontrent pas de ligne politique claire. Par exemple, ils n'établissent pas de bilan des démarches participatives et ne peuvent donc pas attester d'une plus grande mixité sociale et d'une interaction plus forte au sein des ateliers. Or, nous avons constaté que la catégorie sociale des diplômés était surreprésentée par rapport à l'ensemble (avec beaucoup de retraités de l'éducation nationale). La relative longévité des dispositifs n'est donc pas corrélative à une réduction des inégalités d'accès.

Une des raisons de cette longévité est que les dispositifs participatifs constituent une opportunité pour les collectivités de tisser des partenariats et de mutualiser leurs moyens. Dans la logique actuelle de synergie des compétences des collectivités et de dynamique public/privé, intéresser et fédérer le plus d'acteurs différents devient un enjeu majeur, comme nous renseignait un conseiller en communication qui travaille au service de collectivités territoriales :

Plus c'est visible, plus tout le monde en parle et plus il y a d'impact. On croit davantage les acteurs sur le terrain. II faut savoir rassembler dans un projet, si tu le fais ton action marchera d'autant plus. J'agis en permanence à faire se rencontrer les gens qui peuvent faire progresser les choses, à reconnaître ceux qui peuvent travailler ensemble et qui peuvent se compléter, c'est du lobbying ${ }^{15}$.

Pour les collectivités, les collaborations sont bénéfiques en termes de visibilité. Il y a donc un enjeu marketing important.

En insistant sur les termes " innovation », " créativité » et " expérimentation ", les praticiens montrent un désir d'associer la mise en place de formats participatifs à une démarche de recherche qui se veut plus réflexive et collaborative, et qu'ils justifient au travers de partenariats avec l'université, comme les

13 Une dizaine de personnes, comprenant des artistes, des étudiants (en géographie, en communication et en philosophie), des salariés de la structure culturelle, des urbanistes et des représentants d'associations.

14 Expression reprise par les organisateurs dans les programmes de la Biennale arts-sciences.

15 Discussion informelle avec un professionnel de la communication intervenant auprès de collectivités territoriales, janvier 2018. 
travaux sur les dispositifs participatifs le démontrent : " Bien loin de chercher à instrumentaliser les chercheurs en sciences sociales, nombre de praticiens de la participation seraient au contraire intéressés par leur travail critique. Ce qui renvoie aussi à une autre caractéristique du monde social de la participation : la porosité des frontières " (Blondiaux et al., 2016). Cette demande sociale exprime une recherche de légitimation que les professionnels éprouvent dans la caution intellectuelle de ces partenariats.

Une caution intellectuelle qui permet également de justifier l'aspect improvisé des dispositifs participatifs. C'est au moyen de mots-clés et de photos apportées par les participants que les animateurs accompagnent l'écriture de scénarios sur les usages du territoire. Il est important de noter que la restitution délivrée en public est construite à partir de fragments de récits et de beaucoup d'improvisation. Il est encore plus marquant de voir à quel point cette part d'improvisation est assumée par les services qui organisent les ateliers :

Au début, même si on n'est pas sur des scénarios ou des histoires, ce qui peut être un peu difficile des fois pour eux parce qu'il faut se mettre dedans... et bien ils auront les deux intervenants qui seront beaucoup là-dessus, sur l'eau et le territoire finalement, l'eau dans l'agglomération, etc. Donc du coup, après on peut leur proposer de reformuler en lien avec des photos, l'eau et la ville, l'eau et le territoire, et on peut tirer un fil comme ça. Déjà je trouve que rien que ça, effectivement on pourrait l'écrire, ça pourrait déjà être une matière, qu'après on scénarisera plus ou moins avec les photos ${ }^{16}$.

Les animateurs s'appuient sur des « compétences " (Pichon, 2009) scéniques davantage que sur l'expression orale des participants. Or, celles-ci nécessitent tout autant de savoir-faire, et par conséquent d'entraînement. Les participants ont d'ailleurs suivi une préparation avec l'animateur sur quelques séances, ce qui démontre que l'exercice n'est pas aisé. Formuler des sensations, se mettre en mouvement, prendre des photos et réaliser des vidéos des situations ne vont pas de soi. La mise en scène implique d'être à l'aise avec son image et son corps. En effet, certains participants ont exprimé de la réticence à l'annonce des restitutions en public et ne sont pas revenus à l'atelier suivant. En revanche, les plus investis se sont montrés de plus en plus motivés au fil des ateliers du fait que les productions prenaient une tournure scénique. Les contraintes de production sont donc fortes et clivantes : produire des récits des usages, se mettre en scène, définir des protocoles de réalisation, adresser un discours aux experts... Dans l'ensemble, cette productivité est moins bien perçue par les participants qui sont aujourd'hui mieux exercés aux dispositifs participatifs et moins dupes quant à leurs objectifs quantitatifs. 


\section{2. \\ Captation sensorielle du territoire}

L'engagement est rendu visible avec les parcours sur le territoire et il est rendu visuel avec la scénarisation des récits des parcours. L'engagement corporel n'est donc pas mis sur le même plan que l'argumentation verbale qui fut le modèle prédominant des débats publics. Le faire supplante le dire, comme si le faire était moins clivant que le dire et qu'il permettait de mieux lutter contre les inégalités d'accès à la parole :

Et donc l'objectif des AMC \{Ateliers Métro Citoyens\} par rapport aux Jeudis du projet de l'agglo c'est justement d'aller plus sur le terrain, dans la proximité par rapport aux gens, d'aller voir d'autres gens qui ne se déplacent pas sur cette forme de débat. Donc du coup, c'est de leur proposer aussi d'autres possibilités d'accès à la parole que la conférence-débat avec le micro qui circule dans un amphi ${ }^{17}$.

« D'autres possibilités d'accès à la parole » dénote un amalgame entre démocratisation culturelle et démocratie culturelle. Cet amalgame s'enracine dans une métonymie qui consiste à confondre les outils de l'action théâtrale avec la participation elle-même, autrement dit, la scénarisation avec l'accès à la parole. Tels sont les enjeux en présence de la participation : comment aller chercher les publics éloignés des lieux de discussion et quels modes d'expression leur autoriser? Dans nos cas, une délégation de l'expression " collective " semble ciblée à travers le truchement de la mise en scène théâtrale. Il est question de faire se sentir membre agissant d'une communauté, comme l'ont développé les théoriciens du théâtre : "Par cette délégation implicite, la salle [...] manifeste une curiosité sur elle-même, un élan de retour collectif sur soi, un désir d'examen. L'agissant ou l'acteur se pensant comme venant de la salle, est mû par l'aspiration collective qui le pousse vers la scène » (Cormann, 2012, 37). Le recours à la configuration théâtrale - salle/scène - pour les restitutions exprime donc une croyance en une libération magique de la parole de participant.

Plus encore, il y a une vision de l'engagement corporel et du cadre théâtral qui agirait en tant que " caution citoyenne ». Le jeu sur scène et les mises en scène des participants dans l'espace public (comme par exemple la séance de pilâtes au centre d'art contemporain d'un groupe) sollicitent la fonction scopique, avec une dialectique entre "regardant " et "regardé », suivant la chercheuse Chantal Guinebault-Szlamowicz : "Les frontières dans l'espace du théâtre mettent en place les rapports regardants/regardé, distance/proximité, intérieur/extérieur. Le corps est donc en jeu, autant que le regard » (Guinebault-Szlamowicz, 2011, 98-99). Dans une conception d'un espace de confron-

17 Entretien d'approfondissement réalisé avec la chargée de mission Prospective et participation des habitants, le 5 juillet 2012. 
tation entre regardants et regardés (ici le public habituel et les participants eux-mêmes), le théoricien de théâtre Christian Biet envisage la scène comme un « processus sensible et cognitif d'oscillation générale ». En le transposant ainsi aux situations de discussions publiques, la scène devient alors un lieu d'interrogation des positions, " où les regardants comme les regardés viennent, chacun leur tour, ou ensemble, interroger dans la séance ce que leurs positions, ce que leur pensée et ce que leurs propositions signifient » (Biet, 2013, 93). Cette configuration théâtrale porte en elle un imaginaire d'émancipation du public. En étant mis en scène, l'habitant deviendrait agissant aux yeux des personnes du public.

Or, en faisant faire aux participants des performances urbaines afin qu'ils recueillent des matériaux sensibles à restituer en public, les concepteurs/animateurs naturalisent une expression sensorielle et sensitive fondée sur les cinq sens. L'utilisation de la sensorialité fait désormais partie des stratégies marketing des organisations (Quinton, 2002), très présentes dans la culture (Bourgeon-Renault, 2014). Ces modes traduisent une volonté des collectivités de matérialiser et d'illustrer des liens sensibles avec le territoire en vue de mieux faire adhérer la population aux débats publics :

Oui parce que quand même, mine de rien, nos Ateliers Métro-Citoyens, c'est tout à fait intéressant de faire bosser les gens mais il faut les faire travailler en lien avec le territoire. Donc il faut quand même que le récit... c'est pour ça que moi je me disais on pourrait peut-être leur donner quelques mots clés, qu'on ne parte pas... on ne fait pas de la littérature. Donc l'objectif c'est quand même de produire leur vécu par rapport à l'eau, dans l'endroit où ils vivent, donc dans la ville, dans le territoire ${ }^{18}$.

Les déambulations dans la ville sont dès lors mises en place pour récolter des preuves " organiques " afin de prouver l'existence d'une vitalité du territoire. Ces matériaux organiques servent à produire des éléments concrets spécifiques pour appuyer une présence incarnée des usagers. Si le but est de favoriser une identification affective au territoire, l'effet constaté est de détourner de la discussion, confirmant ainsi la thèse de Magali Nonjon, d'une stratégie qui consiste "soit à déporter le dispositif en dehors de son arène de débat habituelle, soit à centrer le dispositif sur une production concrète et donc, ce faisant, à suspendre le débat pour confronter les participants à la réalité des choses " (Nonjon, 2005). Pour autant, ces éléments sont toujours restitués dans des espaces cloisonnés et sous une autorité institutionnelle (un lieu du patrimoine historique pour la Métro et l'institution culturelle elle-même pour le dispositif sur le circuit-court). Scénariser les usages du territoire permet aux collectivités de faire partie d'un territoire communicant au sein duquel 
des formats de débat sont testés, mais où la conflictualité n'est pas prise en charge. Les problèmes sont bien illustrés cependant ils ne sont pas véritablement discutés. En plaçant les usagers en tant qu'agissants, cela permet de détourner l'attention du débat. Cette parade agit en diluant la responsabilité, avec l'impression que celle-ci est partagée par tous les acteurs, comme dans l'exemple que nous allons développer.

\section{3. \\ Un territoire de consensus}

Ces ateliers ouverts n'ont pas pour vocation de trancher une question. Ils n'ont pas non plus pour fonction principale la gestion des conflits, comme ce peut être le cas des dispositifs de Commission nationale du débat public ${ }^{19}$. L'important est que les participants produisent des matériaux exploitables par les services - document écrit ou restitution orale. Les élus locaux et les dirigeants de structures vont s'appuyer sur ces matériaux pour mieux s'adresser à leurs cibles et valoriser leurs partenariats ${ }^{20}$, comme ces extraits d'entretiens le rapportent :

Après, on a tellement peur du débat polémique, on a tellement peur de ne pas maîtriser les choses, on a tellement peur de tout ça, qu'aujourd'hui on n'y va pas très clairement. Comme on est du coup sur des sujets aujourd'hui qui ne sont pas polémiques, du coup la contrainte elle est descellée. Du coup, c'est plutôt facile d'expérimenter, d'aller voir les acteurs. Je n'ai pas de contraintes sur qui aller voir comme acteurs, sur comment monter dans le fond et dans la forme. Donc ce que je me dis, mais après ça c'est dans ma tête, me dire allons-y pour l'instant sur des sujets faciles, ça donne confiance, on apprend à faire, on apprend à faire ensemble. Je pense qu'on a raison et qu'on devrait encore plus aller vers une diversification de types de productions, de créations, et que c'est ça qui va faire la richesse... Mais la demande elle n'est pas écrite comme ça quoi ! Parce qu'en gros aujourd'hui la seule demande, c'est qu'ils (les ateliers citoyens) se fassent, et qu'ils ne fassent pas trop de vagues ${ }^{21}$.

19 Voir les travaux suivants sur les dispositifs participatifs qui ont pour objet la gestion de la conflictualité : Blondiaux, 2009 ; Fourniau, 2007 ; Bobbio et Melé, 2015.

20 À titre d'exemple, la mise en place de plusieurs parcours/déambulations thématiques avec des publics pour la Biennale Arts-Sciences d'octobre 2013 et la création d'un nouvel onglet sur le site de l'institution culturelle « Devenez Acteurs de curiosité territoriale ", qui reprenaient le nom et les grandes orientations définies avec les participants sans qu'ils n'en soient informés.

21 Entretien d'approfondissement réalisé avec la chargée de mission Prospective et participation des habitants organisant les Ateliers métro citoyens, le 5 juillet 2012. 
Le positionnement consensuel est pleinement reconnu, voire même défendu, par les concepteurs/animateurs. Il est tout à fait intéressant de constater que le format n'est que secondaire face au consensus recherché pour fédérer un maximum de participants et de partenaires. La participation des artistes et les formes artistiques qu'ils proposent pour poétiser les récits paraissent dès lors instrumentalisées, puisque le résultat est souhaité lisse et formaté :

Par moment, je trouve que c'est un geste vachement positif de se dire allez on se met dans un consensus et comme ça, il y a un résultat qui est le même pour tout le monde et qui est exploitable. Après, de ce que je connais de mon directeur, je ne pense pas que ça soit pour ce côté-là des formes de restitution, je pense que c'est juste pour faire consensus là, voilà [...]. Après, c'est aussi une première expérience avec l'ensemble de ces partenaires. Moi je trouve que c'était positif d'aller vers quelque chose qui soit plus consensuel, et puis nous, ça peut toujours être exploitable. Ce n'est pas dans le power-point ou le truc de la fin, c'est le fait que les gens ont fait l'expérience, qu'ils se sentent en capacité de la reproduire, ce n'est pas que le document final qui compte. On est parti sur un projet de 3 ans, nous c'est important d'avoir ces partenaires, c'est juste que la mayonnaise n'a pas pris comme il fallait. Je pense qu'on n'a pas envie de les perdre, on a envie de continuer avec eux et que par moment, on est un peu obligé de faire consensus ${ }^{22}$.

À titre d'exemple d'évitement d'un conflit par la scénarisation, lors de la restitution publique sur le thème de l'eau, une mésentente est survenue entre les participants et les experts autour des termes «taxe " et " redevance ». Une participante avait préparé une intervention avec une facture d'eau afin d'obtenir des précisions sur les différentes taxes qu'elle payait (régie, syndicats, etc.). C'est au travers d'un jeu de rôle préparé en amont avec un autre participant qu'elle a interpelé les spécialistes présents sur le manque de clarté des taxes, ainsi que sur le manque d'information à propos des compétences des différents services gestionnaires en matière de distribution et d'assainissement des eaux. Sa question n'a pas obtenu de réponse précise au moment de cet échange. Elle fut par ailleurs accusée par les spécialistes de ne pas avoir "étudié suffisamment son sujet " et fut priée de revoir son utilisation du terme " taxe " au lieu de « redevance ", sous-entendant qu'elle n'avait pas bien lu sa facture.

Par conséquent, en axant le débat sur des éléments de langage, les responsables ont évité de détailler les calculs. Quelques mois plus tard, nous avons intercepté un article dans le magazine de la communauté d'agglomération qui traitait des services de l'eau potable. L'article comportait une infographie de facture d'eau simplifiée, n'expliquant toujours pas la situation de l'usager. Les 
communicants ont ainsi saisi l'opportunité d'utiliser le récit des participants. Sauf qu'encore une fois, il s'agit plus d'une mise en forme de l'information que d'une mise en perspective. Cette stratégie va avoir comme effet de lisser la conflictualité : les professionnels de la participation peuvent subtilement faire focaliser l'attention sur la mise en scène de l'expérience et conduire à amalgamer la forme (le jeu et ses supports) et le contenu (les politiques locales), en renvoyant le discours des participants à sa mise en scène. C'est un moyen de disqualifier les participants et de ne pas leur apporter de réponses précises.

À défaut d'établir un dialogue fondé sur l'explication et la recherche de solutions, la mise en scène des usages du territoire fait donc ressortir des points de tension. La scénarisation des usages par les participants permet à la fois de révéler et de reporter l'attention sur la forme plutôt que sur les contradictions de la communication publique. S'agissant des contradictions sur les formats eux-mêmes, les organisateurs ont fait marche arrière dans la manière d'envisager la restitution. Au dernier moment, ils ont donné des indications pour que la production puisse être exploitable ${ }^{23}$ par la suite, ce qui traduit une visée purement instrumentale :

Tu vois c'est ce que je te disais tout à l'heure, on ne va pas faire de la littérature, même si de la littérature, on pourrait en faire des choses tout à fait intéressantes. Si on veut que ça rentre assez rapidement dans le fonctionnement de la structure, il faut qu'on fasse le lien tout de suite avec ce que fabrique l'institution. Et c'est ça la difficulté. N'empêche que j'entends bien à l'endroit où je suis, à la Métro que... comment dire... voilà que... " t'es gentille avec tes trucs que tu produis m'enfin bon... ", voilà tu vois ${ }^{24}$ ! Il y avait un côté très bien fait sur l'attendu, qui était scolaire, mais peut-être que l'idée, c'était d'inventer autre chose. Ça veut dire que la prochaine fois si on recommence, il faut rediscuter en disant " est-ce qu'on se met tous d'accord que le rendu il a telle forme », ou alors, tout à coup on se dit que parce qu'il y a une expérience, l'idée c'est que chacun peut la restituer comme il le veut. Les gens étaient déstabilisés parce qu'ils n'étaient pas sûrs de ce qu'il fallait faire. Après,

23 Pour l'atelier de l'institution culturelle, un mail a été adressé à l'ensemble des participants la veille de la restitution en public précisant que le rendu devait permettre de faire une évaluation des pistes proposées : «Afin que nous puissions avoir un moment d'échange intéressant en confrontant nos démarches et productions et afin de conserver une trace de la démarche, il est demandé que la restitution soit construite sous forme d'un power-point organisé selon la grille suivante : 0 . Présentation de l'équipe, 1 . Compréhension de la question, 2. Protocole développé, 3. Matériel collecté, 4. Synthèse thématique, 5 . Synthèse sur la démarche, 6 . Eléments et questions pour le débat. Textes, images, chansons, vidéos, danses, cuisine, dessin [...] : toutes les formes de restitutions sont possibles mais devront s'intégrer dans la grille ci-dessous qui permettra les comparaisons et dialogues ".

24 Réunion de préparation avec la chargée de mission Prospective et participation des habitants organisant les Ateliers métro citoyens, le 6 avril 2012. 
tout ça a généré des choses différentes, qui pouvaient être ressenties comme discordantes. Après, ce que je pense, c'est que si on n'est pas d'accord sur ce qu'on veut comme résultat, et que les résultats n'ont pas les mêmes formes, ils sont moins utilisables, c'est tout ${ }^{25}$.

En somme, le contenu est pensé par les politiques et les dirigeants sous une approche plus managériale que communicationnelle. La collectivité intervient sur la scénographie (versant marketing) et sur la mise en relation des différents acteurs (versant managérial), faisant ainsi se confondre la participation et la représentation.

\section{4. Le dépassement des contraintes}

Les temps d'argumentation verbale sont de plus en plus réduits au profit d'une communication davantage centrée sur l'image, avec des mises en scène et des productions qui viennent remplacer les descriptions et les explications. II y a un décentrement de la parole au profit de l'activité expérientielle de l'usager. La scénarisation démontre qu'il y a une volonté de styliser l'acte de participer, de le rendre plus esthétique, plus spectaculaire. Pour autant, l'expérience scénique a été un moteur pour des participants qui au départ ne souhaitaient pas prendre la parole en public. Comme le soulignent les chercheuses en sciences de l'information et de la communication Julia Bonaccorsi et Virginie Julliard pour les débats publics, «le dispositif est également produit par les actions qu'il rend possibles sans qu'elles n'aient été envisagées dans son projet » $(2012,243)$. Les participants se sont en effet emparés de la mise en scène afin d'exprimer leur point de vue sur les cadres imposés : en pointant par leur jeu scénique le manque d'information sur les sujets proposés, l'imprécision du cadre de la participation, la confusion et les contradictions des énoncés, et l'absence d'objectifs clairs de participation. Le plus étonnant est que la scénarisation a permis un réel dépassement de soi chez certains. Dans les entretiens, plusieurs d'entre eux avaient exclu toute possibilité de faire de la politique une activité régulière ou une carrière, ayant éprouvé de la difficulté avec la prise de parole en public. Pourtant, quelques-uns se sont ensuite présentés sur des listes électorales et ont obtenu un siège d'élu à la municipalité. L'atelier a par conséquent présenté une opportunité pour eux de se lancer dans la politique locale. Restituer publiquement l'expérience sous forme théâtrale a donc donné accès à une puissance expressive politique, et la possibilité de réaffirmer l'idée que le débat public nécessitait une formation à la délibération.

25 Entretien d'approfondissement réalisé avec la responsable du Festival ArtsSciences de la Scène nationale arts-science l'Hexagone de Meylan organisant les Rencontres de l'Imaginaire, le 7 mars 2013. 
Ce faisant, ces pratiques de participation n'inscrivent pas seulement le participant dans une " activité dramaturgique », en opérant une stylisation de son expérience suivant la conception du philosophe allemand Jürgen Habermas :

Dans l'activité dramaturgique, les intéressés exploitent ce fait et commandent leur interaction en réglant l'accès réciproque à la subjectivité de chacun. C'est pourquoi le concept central de la représentation (ou de l'expression) de soi ne désigne pas une conduite d'expression spontanée, mais la stylisation de l'expression d'expériences que l'on a faites soi-même, en fonction des spectateurs (2005, 422).

En se mettant en scène, l'usager montre également le lien symbolique qu'il construit avec l'institution. Il démontre qu'il agit sur la relation en la politisant, ici par le truchement de la scène. La performance collective donne à voir les conditions de la prise de parole de l'usager au sein de l'institution. Elle devient une tactique - de contourner la scène de la représentation avec sa logique productiviste - pour se réapproprier une place d'acteur du territoire. Monter sur scène et produire un récit de la participation complexifie l'espace intersubjectif. Se produire sur scène et jouer les usages de son territoire institue une relation qui met en tension la production d'un discours d'usager, sa performativité et son exploitation par l'institution. La mise en scène permet ainsi de faire émerger les paradoxes, dans les stratégies et tactiques des dispositifs participatifs - souhaités performants et accrocheurs par les responsables, formalistes et expérimentaux par les animateurs et informatifs et formateurs par les participants. 
Bibliographie

Aubouin N., Kletz F., Lenay O. (2010). "Médiation culturelle : l'enjeu de la gestion des ressources humaines ". In Culture études, vol. 1, Paris, DEPS, Ministère de la Culture et de la Communication, p. 1-12.

Appel V., Bando C., Boulanger H., Crenn G., Croissant V., Toullec B. (2008). La mise en culture des territoires, Nouvelles formes de culture évènementielle et initiatives des collectivités locales, Nancy, Presses universitaires de Nancy.

Berger M. (2008). «Répondre en citoyen ordinaire. Pour une étude ethnopragmatique des engagements profanes ». In Tracés, Revue de Sciences humaines, $n^{\circ} 15$, p. 191-208.

Biet C. (2013). « Le spectateur/ les spectateurs ". In Théâtre public. Penser le spectateur, $n^{\circ} 208$, avril-juin, p. 89-93.

Blondiaux L. (2009). « Démocratie délibérative VS démocratie agonistique? Le statut du conflit dans les théories et les pratiques de participation contemporaines ". In Carrel M., Neveu C., Ion J. (dir.), Les intermittences de la démocratie : formes d'action et visibilités citoyennes dans la ville. Paris, L'Harmattan, p. 277-290.

Blondiaux L. et al. (2016). «Introduction. Chercheurs et acteurs de la participation : liaisons dangereuses ou collaborations fécondes ? ". In Participations, vol. 3, n 16, p. 5-17.

Bobbio L., Melé P. (dir.) (2015).

Dossier "Conflit et participation, le cas des choix publics territoriaux ". In Participations, vol. 3, n 13, p. 7-171.

Bonaccorsi J., Julliard V. (2012). «Écrire, parler, documenter : enjeux normatifs et pratiques situées de l'expression dans les procédures de débat public ", Communiquer dans un monde de normes. L'information et la communication dans les enjeux contemporains de la « mondialisation », p. 243-256.

Bouquillion P., Pailliart I. (2003). «Techniques d'information et de communication et développement des territoires : vers de nouveaux rapports entre l'état et les collectivités territoriales ? ". In Études de communication, $\mathrm{n}^{\circ} 26$. Disponible sur http://journals. openedition.org/edc/126 (page consultée le 04 décembre 2018).

Caune J. (1981). Esthétique de I'animation culturelle, Grenoble, Université des langues et lettres.

De Certeau M. (1990). L'invention du quotidien. 1. Arts de faire, Paris, Gallimard.

Cormann E. (2012). Ce que seul le théâtre peut dire. Considérations poélitiques, Besançon, Les Solitaires Intempestifs.

Da Lage E. (2008). « “Les ambassadeurs de Lille 2004, capitale européenne de la culture", un 
dispositif politique normatif ? ". In Appel V., Bando C., Boulanger H., Crenn G., Croissant V., Toullec B., La mise en culture des territoires, Nouvelles formes de culture évènementielle et initiatives des collectivités locales, Nancy, Presses universitaires de Nancy.

Dewey J. (2010). Le public et ses problèmes, Paris, Gallimard.

Didier C. (2015). Enjeux communicationnels et injonctions à la créativité dans des dispositifs participatifs, Thèse de doctorat, Grenoble, Université Grenoble Alpes.

Didier C. (2015). « L'institution sous une forme théâtrale : quand les publics mettent en jeu leurs compétences créatives ». In Dalley S., Bendana K., Laouani F. (dir.), Ressources de la créativité. Une expérience franco-tunisienne. Paris, L'Harmattan, p. 175-185.

Didier C., Martin-Juchat F. (2014). «Analyser les conditions d'émergence de la figure de l'habitant à travers la mise en œuvre d'un dispositif de co-construction d'une parole citoyenne ». In Bonnet J., Bonnet R., Raichvarg D. (dir.), Communication et intelligence $d u$ social. Acteurs, auteurs ou spectateurs des médias, de la consommation et des territoires, Tome 1. Paris, L'Harmattan, p. 145-162.

Fourniau J.-M. (2007). « L'expérience démocratique des "citoyens en tant que riverains" dans les conflits d'aménagement ». In Revue eu- ropéenne des sciences sociales, $X L V, n^{\circ} 136$, février 2007, Genève, Droz, p. 149-179. Disponible sur : http://ress.revues.org/95 (page consultée le 04 décembre 2018).

Goffman E. (1987). Façons de parler, Paris, les Éditions de Minuit.

Goffman E. (1973). La Présentation de soi. La Mise en scène de la vie quotidienne, Paris, les Éditions de Minuit.

Guinebault-Szlamowicz C. (2011). "La scénographie comme dispositif : le cadre au théâtre (image, espace et lieu) ». In Bardin C., Lahuerta C., Méon J.-M., Dispositifs artistiques et culturels. Création, institution, public. Lormont, Le bord de l'eau, p. 82-100.

Habermas J. (2005). Logique des sciences sociales et autres essais, Paris, PUF.

Le Bart C., Lefebvre R. (dir.) (2005). La proximité en politique. Usages, rhétoriques, pratiques, Rennes, Presses universitaires de Rennes.

Lefebvre R. (2005). « La proximité à distance. Typologie des interactions élus-citoyens $»$. In Le Bart C., Lefebvre R., La proximité en politique. Usages, rhétoriques, pratiques. Rennes, Presses universitaires de Rennes, p. 103-127.

Lyotard J.-F. (1980). Des dispositifs pulsionnels, Paris, Christian Bourgois Editeur.

Monnoyer-Smith L. (2006). «Être créatif sous la contrainte. Une ana- 
lyse des formes nouvelles de la délibération publique. Le cas DUCSAI ». In Politix, vol. 3, n 75, p. 75-101.

Monnoyer-Smith L. (2007). Les voies de l'expression citoyenne dans les sociétés modernes : formes et contraintes des dispositifs du débat public, Mémoire d'habilitation à diriger des recherches, Université de Technologie de Compiègne.

Mourin G. (2002). « Le Théâtre-action ». In Pensée plurielle, vol. $n^{\circ} 4, n^{\circ} 1$, p. 21-26.

Nonjon M. (2005). « Les usages de la proximité dans le champ participatif ». In Le Bart C., Lefebvre R. (dir.), La proximité en politique : usages, rhétoriques, pratiques. Rennes, Presses universitaires de Rennes, p. 169-182.

Pailliart I. (1993). Les territoires de la communication, Grenoble, Presses universitaires de Grenoble.

Pichon P. (2009). « La prise en compte des compétences des habitants et des usagers dans les projets urbains ". In Carrel M., Neveu C., Ion J. (dir.), Les intermittences de la démocratie : formes d'action et visibilités citoyennes dans la ville. Paris, L'Harmattan, p. 185-193.

Quinton P. (2002). «Stratégies esthétiques de l'organisation et pragmatique du sensible ». In Recherches en communication, $n^{\circ} 15$, p. 77-93.

Ricoeur P. (2005). Parcours de la reconnaissance : trois études, Paris, Seuil. 